\title{
A Design of Framework for AGRI-CLOUD
}

\author{
K.Venkataramana ${ }^{1}$, Dr.M.Padmavathamma ${ }^{2}$ \\ ${ }^{1}$ Department of Computer Science, S.V.University, Tirupati, A.P, India, \\ ${ }^{2}$ Head, Department of Computer Science, S.V.University, Tirupati, A.P, India,
}

\begin{abstract}
Information Technology is used in various fields like education, business, Medicine, Weather forecasting systems, Communications etc., but not used vastly in Agriculture sector which is main livelihood for majority of people in rural areas and which also drives economy in developing countries like India, China, Brazil etc., and also over $45 \%$ of economy is contributed by Agriculture sector in these countries. Most of the people living in rural areas will depends on agriculture does not have facilities or cost may be very high to technology to Agriculture sector to yield good results. In this paper we design and develop AGRI-CLOUD which provides assistance to the farmers in analyzing crop diseases, getting required suggestions and finding appropriate fertilizers during cultivation at minimum cost from experts at research stations or from Government officials in Agriculture departments in a easily understandable natural languages. We design this cloud model to benefit various stake holders in agriculture field to provide precise and accurate information along with various security related concepts for providing integrity, security and Authentication for agricultural data shared or stored in cloud data center, along with Agri-Expert Service.
\end{abstract}

Keywords: Agriculture Field, Cloud Computing, IOT, Agri-Data Acquisition, Agri-Expert Service, Agri-Data storage.

\section{Introduction}

Agriculture plays major role directly or indirectly in improving economy of developing countries like India, China, Brazil, Japan etc., In the current era of liberlisation every sector is competitive including agriculture, so as to compete agricultural sector should also use Information technology to achieve maximum benefits. Mostly the rural areas which depend on agriculture are information-poor and lack of facilities to use results of recent technologies. In past the usage of Information technology is only in the hands of some higher sections of society, but in the current world it is in the hands of all at a lower cost and easy to use. Information communication and technology (ICT) plays vital role in bringing latest bulletins regarding weather reports, prices, usage fertilizer, sowing of crops etc., to farmers at rural areas. Now-a-days government is pushing for better technology delivery schemes even to remote rural areas by offering telecom services including internet at cheaper prices which will help farmers to produce quality crops and to compete with markets at international level.

Agriculture sector will have characteristics like Spatiality, Complexity, Enormousness, Dynamics and number of factors that affect production and quality and will be more as it is different for each crop. Because the environment of farmland is a very complicated ecology system and involves many different kinds of factors from environment to humane, from ecology to economic, from geography to society etc., the collecting of data usually incurs the substantial costs and technologies [1]. Recent trends show that IOT (Internet of Things) is playing major role in agriculture digitization in countries like Japan, Israel etc. The impact of IOT has given good results and it is extending into latest technologies like cloud and grid computing. The IOT is a network of Internet enabled objects, together with web services that interact with these objects. Underlying the Internet of Things are technologies such as RPID (radio frequency identification), sensors, and smart phones. The basic idea of the IOT is that virtually every physical thing in this world can also become a computer that is connected to the Internet, which can receive and send from its location.[2]

Recent Information technology revolution Cloud computing which has evolved by making base of ICT, Internet, Web services and other existing technologies. As we know IOT is also part of it we can use this along with new Computing paradigm to the agriculture sector to improve quality and to achieve better crop results by using information precisely. Cloud computing can be viewed as a new paradigm for dynamic and controlled provisioning of sharable computing resources, maintained by state-of-the-art data centers based on network of Virtual Machines running on high powered physical machines. NIST[3] defines Cloud computing whose main

design aim is to provide convenient, on-demand, network access to a shared pool of configurable computing resources (e.g. networks, servers, storage, applications, and services), which can be rapidly provisioned and released with minimal management effort or service provider interactions. Cloud can be deployed in public, private or hybrid models which provides services in various forms like Software as a Service-SaaS Platform as a Service-PaaS and Infrastructure as Service-IaaS at cheaper cost. As most of the agriculture is in rural areas the services should be offered at cheaper price through various devices that they use, 
so the cloud computing better suits for this purpose that's why we are proposing framework for Agri-Cloud which will give solutions to many problems related to agriculture field.

Cloud computing has a characteristics of elasticity, scalability etc., it can support different types devices like mobiles, web browsers, Tablets, or any cheaper devices it can be used by consumers of any category from farmers to agriculture experts etc. Cloud computing data centers allows you to store vast historical data securely on which many complex calculations can be done which is required for agricultural scientists for new inventions. In rural areas if the departments of agriculture gives timely and accurate agricultural information stake holders can make the right decisions, planning the development of farm lands. If the Information technology is improved Agri-cultural science and education Personnel can improve the research capacity and level of education by gathering the latest agricultural information resources and deliver the same to the end users. So in this paper we have proposed Agri-Expert service as part of Agri-cloud which will benefit agriculture experts, Government officials, farmers.

All the above we discussed cannot be achieved without CapEx and OpEx but cloud can provide the solutions to Agriculture sector(stake holders of Agri domain) at a very cheaper cost which will benefit the developing and poor countries. So in this paper we have proposed a Cloud Framework AGRI-CLOUD which is discussed in later sections. The remainder of this paper is organized as follows Section-2 summarizes previous works of cloud computing on agriculture. In Section-3 we have given design of Agri-cloud and its components in cloud architecture. Section- 4 gives operational aspects of Agri-Cloud proposed and Section-5 provides Technical aspects to be used in deploying Agri-Cloud and final section we have given our conclusions along with the future work.

\section{Related Work}

In paper by Janet Kaaya[4] has summarized the importance information in generating and disseminating agriculture technologies, by various types users to improve agriculture production through information technology. Ji-chu Zhao et.al in there paper[12] has elicited IOT technology(RFID, Mobile, Sensor devices, Internet) and its usage to agriculture field. They have proposed IOT for Agriculture Greenhouse production environment measurement and control system which allows customer to monitor remotely.

In his paper [1] Dan Yan-e discussed about characteristics agriculture data and how Agriculture information management system is useful in agriculture field. He has considered IOT to AMIS for information collection, processing and given an idea about Intelligent Agriculture Management information system which facilitates to make decisions regarding crop growth, Fertilization of soil, pricing etc. in more precise manner. In paper regarding Smart cloud computing [5] Qiao Ying,Chen Hao has used IOT along with Cloud computing and proposed Smart Cloud Computing platform which can consume data from various IOT devices. The data can be stored in Cloud data centers and be processed by smart massive servers to make decisions for problems that require. So by considering above facts we have proposed Cloud model for agriculture field which uses both IOT and Cloud computing technology for data acquisition, processing, storing at large quantities in cloud data centers.

In their work by Yifan Bo, Haiyan Wang[6] has analyzed and given idea of how the latest technologies IOT and cloud computing can be used in agriculture and forestry for precise agriculture management. In his paper he pointed problems in cloud computing regarding security,IPV6, data center maintenance etc. In paper Kaas-based intelligent service model in agricultural by Yin Qirui [7] has proposed an agricultural knowledge service model based on Agriculture expert service with a purpose of providing accurate and efficient agricultural knowledge service to users with browser interface. Author has used existing online expert systems to make decisions and restricted only to browser, so in our Agri-cloud model we have used Agri-expert service in PaaS which can be used by any type of application interface like Mobiles, PDA, browsers and allows decision making in precise manner which will helpful to stake holders to cultivate farm lands.

Mitsuyoshi Hori et.al [8] in their publication Application of cloud computing to agriculture and prospect to other fields has given details about cloud established by Fujitsu Ltd for agriculture field and how it delivers services to farmers regarding pricing and selling of crops. It is based on the model Plan-Do-Check-Act. But many issues like decision making on fertilizer usage, crop disease finding, queries related to pricing etc., and security for data in data centers have not been discussed. By keeping above all we have designed AGRI-

CLOUD model providing services at SaaS, PaaS, IaaS. Main purpose of designing our model is make users in rich or poor countries to use at very cheaper cost. It supports different types of devices and ask queries in their native languages to get expertise solutions from Agri-Expert service AGRI-CLOUD. Agri-Security Service provides authentication for experts pushing and pulling data out of cloud and storing encrypted data in storage layer of cloud. In this paper, we propose AGRI-CLOUD model in next sections along with operational and technical aspects. 


\section{Design Of Agri-Cloud Architecture}

Cloud computing and with combination of (Internet of things)IOT offers resources and services at cheaper cost which is essential for farmers working at cultivation lands, so we have proposed this framework of Agri-Cloud which offers expertise service to farmers regarding cultivation of crops, pricing, fertilizers to be used etc. Scientists working at Agriculture research stations can add their discoveries, suggestions regarding modern techniques for cultivation ,usage of fertilizers, can obtain cultivation history of the region etc.,. AgriCloud can benefit Government officials/private organizations by obtaining/inserting information regarding pricing of crops, farming of lands at various places, benefits to be given to farmers for various crops like supplying seeds, fertilizers etc.

Agri-Cloud framework at SaaS layer supports various services to Farmers to interact with cloud by using any cheaper ways or IOT such as Sensors, Mobile devices, Scanners etc., to query for information and access it in no time at free or cost from free services and by paying meager amount for pay services. Agri-Cloud can use existing cloud infrastructures like networks, servers etc., other than the resources discussed below.

The proposed Agri-Cloud framework shown in fig-1 is a layered architecture contains layers like

1) Agri-Data Acquisition layer(ADAL)

2) Agri-Data Processing layer(ADPL)

3) Agri-Data Storage Service layer (ADSSL).

1) Agri-Data Acquisition layer(ADAL) uses Internet and IOT which provides services to be used by farmers, agriculture experts or government officials to add or query data by using their applications service interfaces either through browsers, Tablet PC's, sensor(RFID) device or mobile devices. ADAL is deployed as SaaS in Cloud which provides various interface services to be used by different types of consumers with different devices. ADAL services layer mainly used for agriculture data acquisition and supply solutions to users. Vast data or historical data used for various purposes is stored in Agri-DB.

2)Agri-Data Processing layer(ADPL) is a Data processing layer contains libraries which will accept data in various formats from various devices and converts into uniform format and performs computations on large data sets and reports to consumers of Agri-cloud. Platform as a service encapsulates a layer of software and provides it as a service that can be used to build higher-level services[9]. ADPL is deployed as PaaS in Agri-Cloud which contains libraries or readymade program modules to be used to build high-level agriculture based applications. ADPL provides service contains libraries for Data security, Data Processing, Expert Decision making and Data Reporting.

Further ADPL has divided into following modules

a) Agri-Secure Data Service module

b) Agri-Data Processing service module

c) Agri-Expert Service Module

d) Agri-Solution reporting Service module.

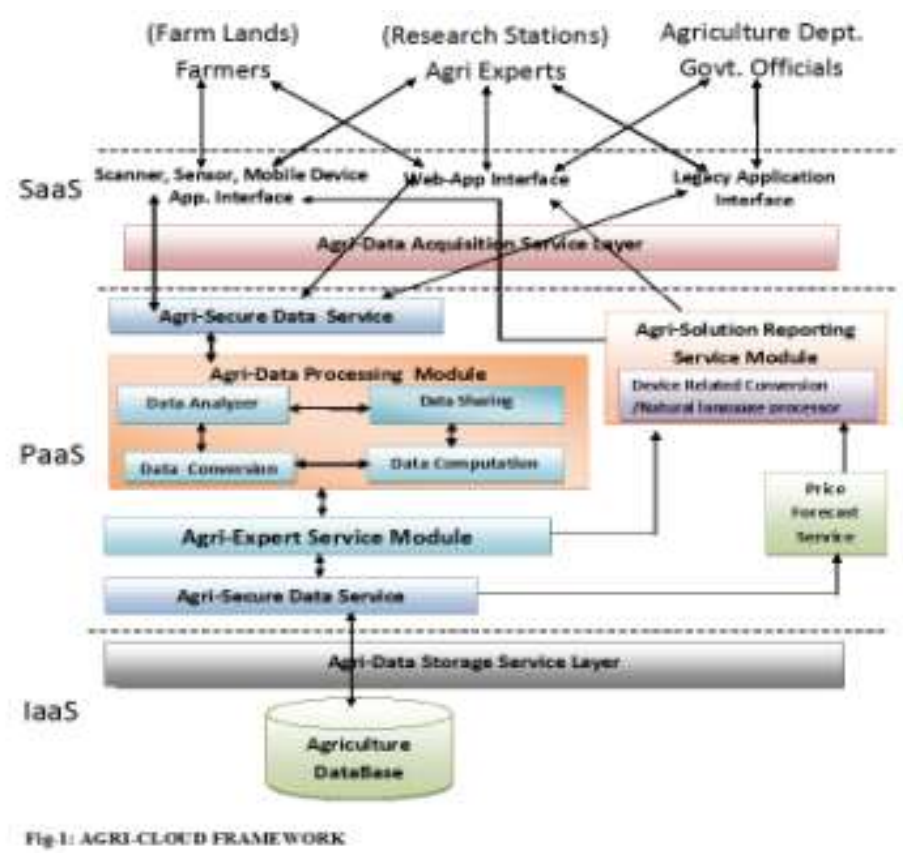


a) ADPL uses Agri-Secure Data Service(ASDS) libraries to provide authentication, integrity, secrecy for incoming data from various sources or reporting them with solutions. Every data source or users are Authenticated by using secure algorithms to access resources of cloud like VM.s[10], databases etc. ASDS deny accessing of malware programs in modifying cloud data or destroying cloud resources deliberately by using agent based IDPS[11].

b) Agri-Data Processing(ADP) service contains libraries for Analyzing the data, Conversion of data from various devices into uniform format Agri-Cloud Data XML (ACDXML), data Computation, natural language processing of data sharing and data sharing if required.

c) Agri-Expert Service (AES) is a expert service layer contains libraries which allows to provide solutions or decisions after processing sensor, image, mobile, query related data from consumers and generates reports sent to ASRSL. ADESL uses various expert service modules which uses fuzzy logic, AI techniques for providing solutions dynamically.

d) Agri-Solution Reporting Service(ASRS) contains libraries which provide reporting service to customers in formats required by them to various devices after conversion to their respective languages by using natural language processor. ASRS will convert data into ACDXML format and it can be consumed by applications in SaaS for reporting users in various ways to them.

3)Agri-Data Storage Service layer (ADSSL) is data storage layer supports database infrastructure facilities to store large amounts of data which is required in agriculture sector for results to be accurate. ADSSL is deployed at IaaS level in cloud which allows data sharing and usage. Agriculture data base contains Agri-Expert knowledge Database(AKDB) contains rules, inferences for decision making purposes, Image Knowledge Database(IKDB) to make decisions based on images received from farmers or consumers from farm lands, Statistical knowledge Database(SKDB) which allows to make decisions regarding amount of land to be cultivated, quantity of seed, fertilizers to be used etc,. Business Knowledge Database(BKDB) contains data to make business related decisions for pricing or for comparisons of business at different locations on different agriculture products. ADSSL uses Agri-Secure Data Service(ASDS) libraries for securing cloud data storage by encrypting it at storage and decrypting during its usage by various services. Encrypted data is stored in AKDB, IKDB, SKDB, IKDB in Agriculture Data base layer for security reasons.

\section{Operational Aspects Of Agri-Cloud}

Agri-Cloud when deployed on cloud will work in layered architecture where layer below will provide services to high level layers. Farmers, Agriculture experts, Government officials etc., will interact with Agricloud by using various devices like Web browsers, Sensors, Mobile devices, PDA etc., will use appropriate application interfaces available at SaaS layer in cloud. SaaS layer will be used as Data Acquisition layer to give input to Agri-Cloud system to update databases by the experts from Agricultural research stations. Farmers at Farm lands can obtain solutions through expert systems available regarding the query given in their natural language.

The PaaS layer in Agri-cloud contains API's to process and analyze data, provides security and authentication to data and its users and delivers expertise solutions through expert service module available. The data from the experts are accepted only through proper authentication provided by the ASDS.ADP service module will process data from experts and converts into unified format and stored as knowledge based inference rules in Agri-Database in IaaS which will be used by expert system to provide solutions to farmers. Data from Experts are classified into Image data, Sensor Data, Statistical data, Rule based data, business related data and stored in appropriate databases in encrypted format by encryption and decryption service provided by ASDS. ADP will perform computations and Sharing of data if require for computation in secure manner.

The main purpose of Agriculture cloud is to provide the solutions to farmers in rural areas for problems related to cultivation at cheaper cost or at free of cost (if funded by government). Agri-cloud uses Agri-Expert system shown in fig-2 which is available as API or libraries or executable code module at PaaS layer to be used by higher layers to provide solutions regarding cultivation or business. AES can be used in various ways like, for example a farmer can send a query through a mobile regarding any cultivation problem and the Intelligent solution provider will direct the query to Agri-Cultivation expert and in turn to Mobile expert module which in turn uses DSS/Inference engine to check the query or rules against the data in $\mathrm{AKDB} / \mathrm{SKDB} / \mathrm{SKDB}$ for required solution and send it back to farmer mobile device by using ASRS.

Similarly for Statistical data or image data. If the disease crop image is send to Agri-Cloud system through any interface it will use Image expert system to find the crop disease from IKDB and sends the name of fertilizer or remedy for the problem in crop in cultivation land. Also farmer or consumer of Agri-cloud service can send a query through a mobile or through any interface in SaaS regarding pricing for crop in particular 
location then the Intelligent solution provider will direct the query to Agri-Business expert which will in turn use DSS/Inference engine to check it in BKDB and reply back to client ASRS in a natural language of the client at that location. AES can provide information regarding amount of fertilizers that they must apply to a particular kind of soil and crop. Government officials at that region/location can educate farmers regarding this by querying the system with soil type and nutrients in soil so that they can suggest quantity and type of fertilizer the farmer can use for that soil in that season. Agri-Cultivation Expert will use inference rules or knowledge in AKDB for providing the solution.

The knowledge acquired from knowledge acquisition layer from domain experts is represented into structured form. There are many approaches for representing knowledge into the knowledge base. Such representation in ESTA is the rule based representation in logical paradigm of simple if-then rules in backward or forward chaining. We have chosen here the backward chaining for knowledge representation with simple ifdo pair in place of if-then rules. These rules are stored in AKDB for fast decision making and achieving solutions to problems from farmers. Here we have considered two major knowledge representations namely Sections and Parameters. The top level of representation of knowledge in ESTA is section. It contains the logical rules that direct the expert system how to solve problem, actions to perform such as giving advice, going to other sections, calling to routines etc. The first section in ESTA is always named as start section. The advice is given when condition(s) in the section is(are) fulfilled. Parameters are used as variable and it determines the flow of control among the sections in the Knowledge Base[2]. Image Expert system will analyze crop disease by analyzing the crop color with the stored high resolution images in IKDB. To process it very highly complex image processing algorithms for clipping, smoothing, segmentation are executed to classify images along with various image attributes and to give final solution. In mobile expert service module Questions or images from mobile is accepted is processed accordingly to report appropriate solutions to the end users.

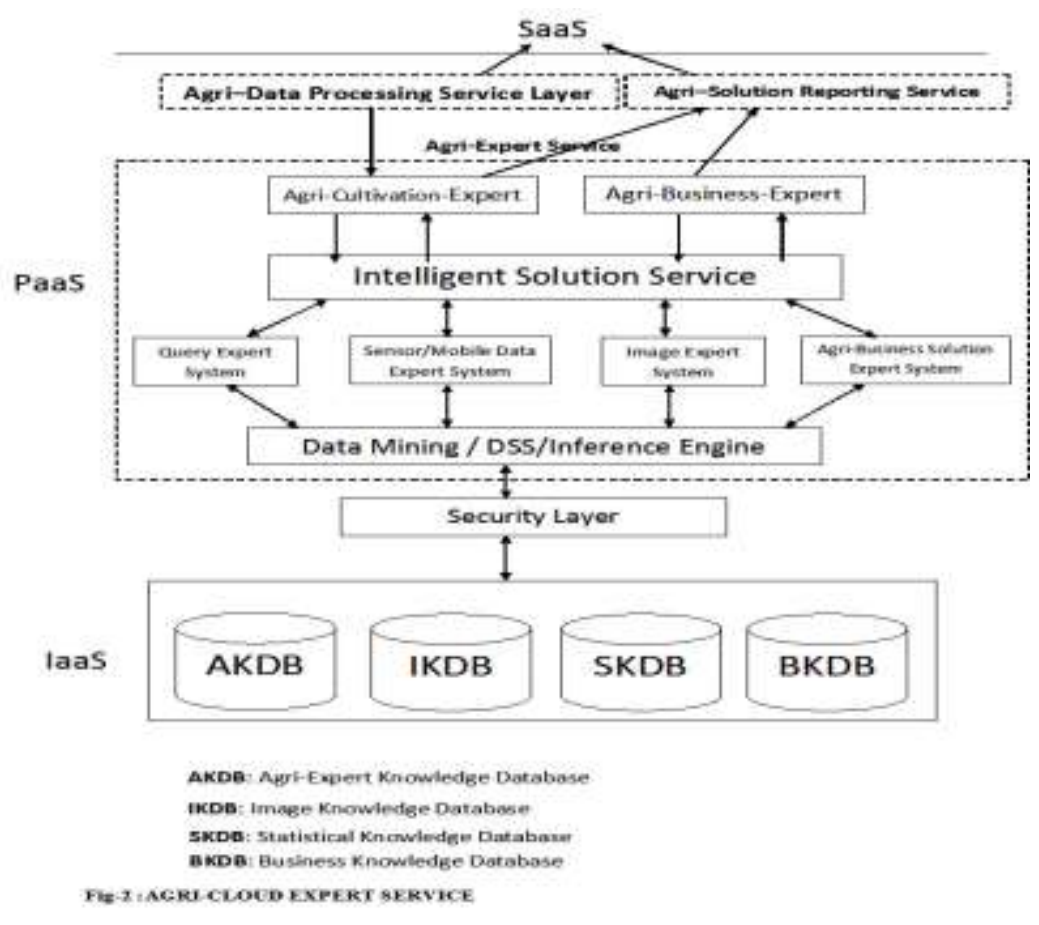

Agri-Data Storage Service layer (ADSSL) which serves as storage layer for agriculture database which is deployed in IaaS layer of Agri-Cloud. The large amounts of agriculture data is stored in databases at cloud data centers in encrypted format for security purposes. The knowledge information to be used by above layers is stored in databases(which in turn tables) AKDB,IKDB,SKDB,BKDB as discussed in above section.

\section{TECHNICAL ASPECTS OF THE AGRI-CLOUD}

We are proposing this AGRI-CLOUD model to be a public cloud/Hybrid cloud to deliver services as discussed in above sections. Our idea is to use cloud open sources like Ubuntu/CentOS, Eucalyptus or in Cloud Foundry or Open Nebula along with Java Platform technologies using MySQL databases for developing the proposed AGRI-CLOUD.The proposed Agri-Cloud can be deployed on machines having on I3/I5 processors with 4 to 8 GB RAM.

Eucalyptus Cloud computing Infrastructure model currently comprises primary services like [13] 
Cloud Controller - which maps to Amazon Elastic Compute Cloud (EC2), allows to deploy and share instance of AGRI-CLOUD Web services, Interfaces to access libraries, API's and data processing layer services like computation, sharing services, security services and controlling data storage facilities for Agri cloud. Front end interfaces can be designed in dot.net or JSP etc.

Storage Controller \& Walrus - which maps to Amazon Elastic Block Storage (EBS) and Amazon Simple Storage Service (S3) which provides data storage layer for AGRI-CLOUD. We can use MySQL database in an instance in an Eucalyptus image. By using Walrus service component we can control or manage AKDB,IKDB, SKDB,BKDB databases proposed in our Model.

\section{CONCLUSION}

In this paper we have proposed Agri-Cloud model along with Agri-Cloud framework with appropriate technical aspects to provide assistance to farmers during crop cultivation to analyze soil, crop cultivation, crop diseases and to give pricing solutions during cultivation in a cheaper means through latest technologies like cloud computing to Farmers, Agriculture Experts, Government officials. An application of cloud computing is more suitable for agriculture as large agriculture data is to be processed and stored at cheaper prices which is essential in developing countries at this juncture. In future we deploy Agri-Cloud by using open source cloud computing technologies as proposed in this paper.

\section{REFERENCES}

[1] Duan Yan-e , "Design of Intelligent Agriculture Management Information System Based on IOT”,IEEE,4 ,.Fourth International erence on Intelligent Computation Technology and Automation,2011

[2] Shikhar Kr. Sarma, Kh. Robindro Singh \& Abhijeet Singh,"An Expert System for diagnosis of diseases in Rice Plant", International Journal of Artificial Intelligence, Volume(1): Issue(1) ,pp 26-31

[3] Recommendations of National Institute of Standards and Technology [online].[availabl http://csrc.nist.gov/publications/nistpubs/800-145/SP800-145.pdf

[4] Janet Kaaya,"Role of information technology in agriculture,"Proceedings of FoA Conference , Volume 4,1999

[5] Qiao Ying,Chen Hao,"The Design of smart cloud computing system" ,International Conference on Computational and Information Sciences,IEEE,2011

[6] Yifan Bo, Haiyan Wang, "The Application of Cloud Computing and The Internet of Things in Agriculture and Forestry", international Joint Conference on Service Sciences, IEEE computer Society,2011

[7] Yin Qirui, "Kaas-based intelligent service model in agricultural expert system", $2^{\text {nd }}$ International conference on consumer electronics, communications and networks,IEEE,2012

[8] Mitsuyoshi Hori, Eiji kawashima, Tomihiro yamazaki, "Application of cloud computing to Agriculture and prospect to other fields",Fijitsu science Technology journal, Volume 46 no.4 pp 446- 454,Oct 2011

[9] Introduction to Cloud Computing architecture, White Paper,1st Edition, June 2009,sun microsystems ,[Online][Available], http://eresearch.wiki .otago .ac.nz /images/7/75/Cloudcomputing.pdf

[10] K.Venkataramana, M.Padmavathama, "Agent Based approach for Authentication in Cloud", IRACST - International Journal of Computer Science and Information Technology \& Security", Vol. 2, No.3, June 2012

[11] K.Venkataramana, M.Padmavathama, "Multi-Agent Intrusion Detection and Prevention System for Cloud Environment " International Journal of Computer Application Volume 49-No.20, July 2012

[12] Ji-chun Zhao, Jun-feng Zhang; Yu Feng ; Jian-xin Guo,"The Study and Application of the lOT Technology in Agriculture", Computer Science and Information Technology (ICCSIT), IEEE, 2010

[13] Eucalyptus: An Open-source Infrastructure for Cloud Computing,[online], http://assets.en .oreilly.com/1/event/45Eucalyptus_The Open Source Infrastructure for Cloud Computing Presentation.pdf 